\title{
Loop Closure Theory in Deriving Linear and Simple Kinematic Model for a 3 DOF Parallel Micromanipulator
}

\author{
Yuen Kuan Yong, Tien-Fu Lu* , Daniel C. Handley \\ School of Mechanical Engineering, The University of Adelaide
}

\begin{abstract}
Various types of micro-motion devices have been developed in the past decade for applications including the manipulation of cells in micro-surgery and the assembly of micro-chips in micro-assembly industries. Most of the micro-motion devices are designed using the compliant mechanism concept, where the devices gain their motions through deflections. In addition, closed-loop parallel structures are normally adopted due to better stiffness and accuracy compared to the serial structures. However, the forward kinematics of parallel structures are complex and non-linear; to solve these equations, a numerical iteration technique has to be employed. This iteration process will increase computational time, which is highly undesirable. This paper presents a method of deriving a simple, linear and yet effective kinematic model based on the loop closure theory and the concept of the pseudo-rigid-body model. This method is illustrated with a 3 DOF (degree-of-freedom) micro-motion device. The results of this linear method are compared with a full kinematic model for the same micro-motion system. It is proved that the derived kinematic model in this paper is accurate and the methodology proposed is effective. The static model of the micro-motion device will also be presented. The uncoupling property of the micro-motion systems, based on the static model, will be briefly discussed.
\end{abstract}

Keywords: Micro-motion systems, compliant mechanisms, linear kinematic model, parallel mechanisms

\section{INTRODUCTION}

In the past decade, micromanipulation has emerged as an important technological advancement. The significance of this advancement is highlighted in its growing applications, such as in the field of micro-surgery, biological cell manipulation and micro-assembly. In micro-surgery, hand tremor is a common problem that reduces the performance of surgeons. It causes cells damage during the operations. The introduction of micromanipulation devices provides a tremor-free surgical environment to the surgeons. Therefore, the success rates of the operations are increased. The advances in microbiology, such as male infertility treatment and cloning technology, have increased the need to manipulate a single cell. This manipulation process is normally referred as biological cell manipulation. Methods, such as embryo pronuclei DNA injection and intracytoplasmic injection (cell injection), are used to introduce genetic material into cells ${ }^{1}$. The conventional cell injection methods, which are conducted manually, require professional training and skills. The manual injection techniques have very low success rates. The micromanipulation systems, which have high positioning accuracies and resolutions, are capable of performing the injection tasks precisely with minimum cell damage and high success rates. In micro-assembly, the sizes of electronics chips have been reduced drastically to micrometers. The integration of all the micro-components into microsystems would be impossible if it was done manually by hand. Therefore, micromanipulation systems are needed to extend human capabilities in the micro-assembly industries.

Different actuation principles have been applied to drive the micromanipulation systems. Piezoelectric (PZT), electrostatic, electromagnetic and shape memory alloy actuators have been utilised to provide fine motion to the systems. However, PZT actuators are the most common driving elements used for the micromanipulation systems due to their high resolution displacements and fast responses.

\footnotetext{
* Corresponding author: tien-fu.lu@adelaide.edu.au; phone +61 8 83033556; fax +61 883034367; School of Mechanical Engineering, the University of Adelaide, North Terrace, Adelaide, SA 5005, Australia;

http://www.mecheng.adelaide.edu.au/robotics/index.html
} 
Most of the micromanipulation systems are designed based on the compliant mechanism concept. Compliant mechanisms generate their motions through elastic deformations whereas conventional rigid-link mechanisms generate their motions via movable joints (e.g. revolute joints). Compliant mechanisms replace most of the joints in rigid mechanisms using flexure hinges. These mechanisms are advantageous over the rigid-link designs in applications requiring micro-motion ${ }^{2}$. Problems such as friction, wear, backlash and lubrications are eliminated. Furthermore, compliant mechanisms have fewer components compared to rigid mechanisms, thus allowing for savings in weight.

Many micromanipulation devices are designed using parallel structure configurations. Parallel structures have closedloop architectures which are advantageous over serial structures. All the actuators of the parallel micromanipulation devices can be located at the base, thus reducing the active mobile mass ${ }^{3}$. Therefore parallel structures have higher loading capacity. Parallel structures also have higher mechanical stiffness, faster manipulation and higher positioning accuracy ${ }^{4}$. These characteristics are beneficial for micro-motion devices.

The micromanipulation system studied in this paper is a three degree-of-freedom (DOF) parallel micro-motion device (also known as $3 \mathrm{RRR}^{*}$ compliant mechanism). It is a monolithic compliant mechanism utilising flexure hinges. The micromanipulation device is actuated by three PZT stack actuators as shown in Fig. 1. It is designed based on the 3RRR mechanism (see Fig. 2). The end-effector platform is attached to the ends of the three linkages as illustrated by the triangle in Fig.1. The end-effector translates along $\mathrm{x}$, y-axis and rotates about the z-axis. This type of parallel compliant mechanism amplifies the motion of the PZT actuators. Its monolithic structure makes the manufacturing process simple and also cost effective. It does not require the assembly of multiple stages to achieve 3 DOF. Therefore, the $3 R R R$ compliant mechanism stage is compact and light.

The 3RRR parallel structure has attracted the attention of many researchers ${ }^{4-7}$. This structure has its advantages, however, the forward/direct kinematics of the parallel mechanism is very complex and non-linear. Although the inverse kinematics of the parallel structures is simpler than that of the forward kinematics, forward kinematics is still required to achieve real-time control of the systems.

The complexity of the forward kinematics is due to the unknown relative motions of the unactuated joints ${ }^{4}$. The motions of the unactuated joints can only be found by solving a set of non-linear equations simultaneously. A numerical iteration technique is normally employed to solve these equations. This iteration process will increase computational time ${ }^{8}$ and hinder controller designs.

This paper presents a method of deriving a simple, linear forward kinematic model of the particular 3RRR parallel micromanipulation device which effectively represents the real-system, and can also be easily calculated in real-time for the implementation of control. The static model of the 3RRR micro-motion system using the results of the derived linear kinematics will also be discussed. The uncoupling property of the 3RRR system will be briefly discussed.
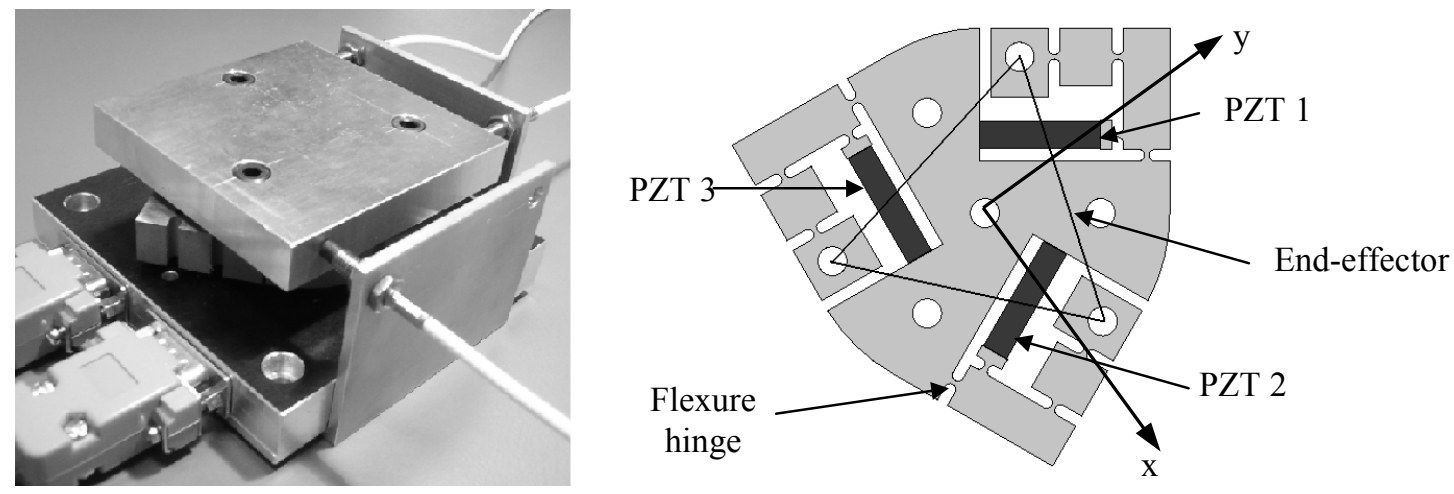

Figure 1: 3RRR compliant micromanipulation device: The micromanipulation system with sensors and end-effector (left); the schematic diagram of the compliant micromanipulation device (right)

${ }^{*} \mathrm{R}$ : Revolute 


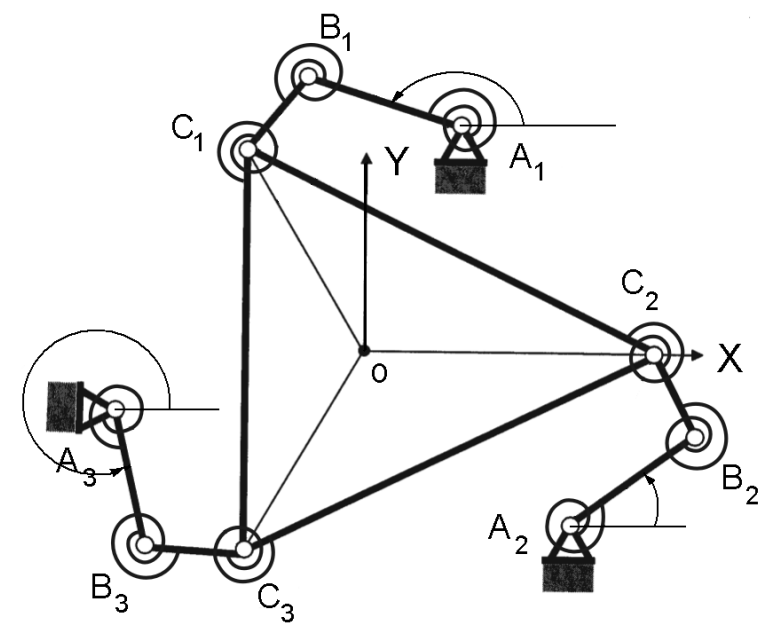

Figure 2: 3RRR compliant mechanism

\section{PREVIOUS WORK}

A few researchers have derived forward and inverse kinematics for parallel manipulators. For macro manipulators, Ma and Angeles have developed an effective method to derive the forward kinematics of a non-compliant 3RRR parallel manipulator ${ }^{4}$. Gosselin and Angeles also developed the kinematics of the same 3RRR parallel manipulator for the purpose of kinematic design optimisation ${ }^{7}$. Tsai et al. developed the forward kinematics of a 3RPS ${ }^{*}$ parallel mechanism where an optimisation technique is used to solve the kinematics ${ }^{9}$. Dunlop and Jones incorporated the concepts of the universal coordinate system and transformation matrices to derive the forward and inverse kinematics of a 3 DOF parallel mechanism ${ }^{10}$. Tahmasebi and Tsai derived direct kinematics of a 6 DOF parallel manipulator ${ }^{11}$. All these methods could possibly be employed to derive the forward kinematics of the micromanipulation systems. However, the derived kinematic equations are complex and non-linear. In addition, all the forward kinematics aforementioned require numerical techniques to solve and this is time consuming.

A number of researchers have also derived the kinematics for micromanipulation systems. Zhang et al. developed a constant-Jacobian method for the kinematics of a $3 \mathrm{DOF}$ micro-motion stage ${ }^{12}$. Ryu et al. derived an inverse kinematic model of a micro-motion stage with $3 \mathrm{DOF}^{13}$. However, the forward kinematics is not discussed. Ohya et al. developed a 3RPS parallel mechanism for micromanipulation ${ }^{14}$. Chung et al. developed a similar spatial 3RPS micromanipulator for tele-operation ${ }^{15}$. Both of these papers discuss only the inverse kinematics but not the direct kinematics. Hesselbach et al. have derived both the direct and inverse kinematics for a 3RRR parallel micro-motion system ${ }^{16}$. Zou has derived a full forward kinematic model for a similar 3RRR structure ${ }^{17}$. However, the forward kinematics are non-linear. The nonlinearities of the kinematic equations require iteration techniques to solve. Shim et al. analysed the kinematics of a 6 DOF parallel manipulator for micropositioning ${ }^{18}$. They presented the methods of deriving the forward kinematics but the equations are again non-linear. The Newton-Raphson method is used to solve the non-linear equations. Although this iteration method is simple and its solutions converge fast, this method is very sensitive to the initial values used. Improper initial values chosen will increase the calculation time. Her and Chang have developed a linear scheme for the displacement analysis of the micropositioning stages ${ }^{8}$. All the geometrically constrained equations are linear and can be solved directly. The linear scheme is demonstrated using single-loop and two-loop stage structures. The displacements of each flexure hinge in the structures, calculated using the linear scheme, are presented. The hinge displacement results are claimed to be accurate. However, the forward and inverse kinematics of the stages are not discussed. The relationship between the actuator displacements and the end-effector displacements/orientations is not considered.

There has been lack of discussion about the benefits of the linearity and simplicity of the kinematics for micromanipulation systems. Therefore, it is of great interest to the authors to develop a method of deriving the simple

${ }^{*}$ R: Revolute, P: Prismatic, S: Spherical 
and linear kinematics, in this case, for a 3RRR micromanipulation system. As aforementioned, linear and simple kinematics does not require iteration technique to solve, hence it reduces the computational time. Furthermore, the linear method presented in this paper is simpler than the conventional way of deriving the forward kinematics. The linear and simple kinematics will also benefit the optimisation designs, where less calculation time is required to evaluate many different trial designs to meet certain specifications.

\section{LINEAR KINEMATIC MODEL}

The 3RRR micromanipulation system shown in Fig. 1 is studied in this paper. Two concepts are employed to derive the linear kinematics for this micro-motion device, namely the pseudo-rigid-body model and the loop-closure theory.

\subsection{Pseudo-rigid-body model}

The pseudo-rigid-body (PRB) model is used to model the deflections of the flexible members using conventional rigidlink mechanism theory ${ }^{2}$. The PRB model assumes that the flexure hinges in the structure act like revolute joints with torsional springs attached to it (see Fig. 3). The other parts of the structure are assumed to be rigid. Therefore, the PRB model is referred as a bridge connecting the rigid-link mechanisms and the compliant mechanisms ${ }^{2}$. The PRB model of the 3RRR compliant mechanism is illustrated in Fig. 3. The flexure hinges are labelled as $A_{i}, B_{i}$, and $C_{i}$, where $i=1,2,3$. $\theta_{A i}, \theta_{B i}$ and $\theta_{C i}$ are the initial angular displacements of the flexure hinges, measured from x-axis. $\Delta \theta_{A i}, \Delta \theta_{B i}$ and $\Delta \theta_{C i}$ represent the small angular displacement increments of the flexure hinges.
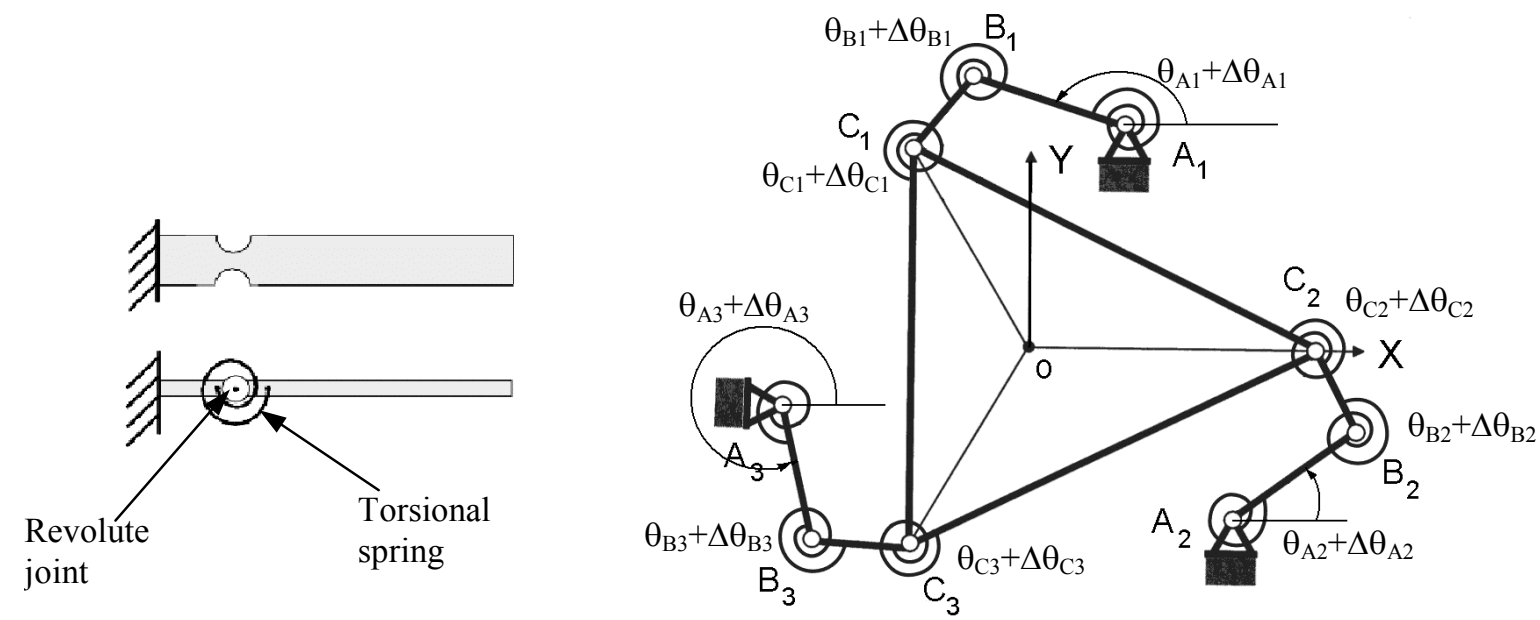

Figure 3: PRB model - single link (left) and the 3RRR compliant mechanism (right)

\subsection{Loop-closure theory}

Loop-closure theory incorporates the complex number method to model a mechanism. For each closed-loop in the mechanism, a loop equation is generated ${ }^{19}$. This equation can be expressed in terms of its real and imaginary parts, resulting in two equations per loop ${ }^{2}$. Unknowns can be found by solving these equations simultaneously.

Complex numbers are used to represent vectors in each closed-loop. The complex number is written as:

$$
Z=r e^{i \theta}=r(\cos \theta+i \sin \theta)
$$

where $\quad r$ is the link length

$\theta$ is the angular displacement describing the initial orientations of the link (see Fig. 4) 


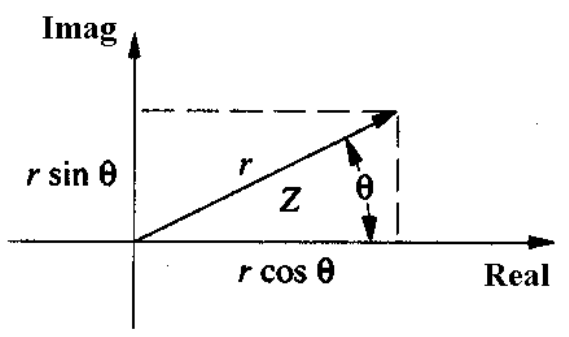

Figure 4: Complex plane

\subsection{Linear forward kinematics}

In Fig. 2, all the flexure hinges labelled $A_{i}$ are actuated (active joints). Flexure hinges $B_{i}$ and $C_{i}$ are unactuated (passive joints). Therefore, $\Delta \theta_{B i}$ and $\Delta \theta_{C i}(i=1,2,3)$ are unknowns. In order to solve these six unknowns, three closed-loops are generated as shown in Fig. 5. Using the loop-closure theory, six equations are obtained from the three loop equations:

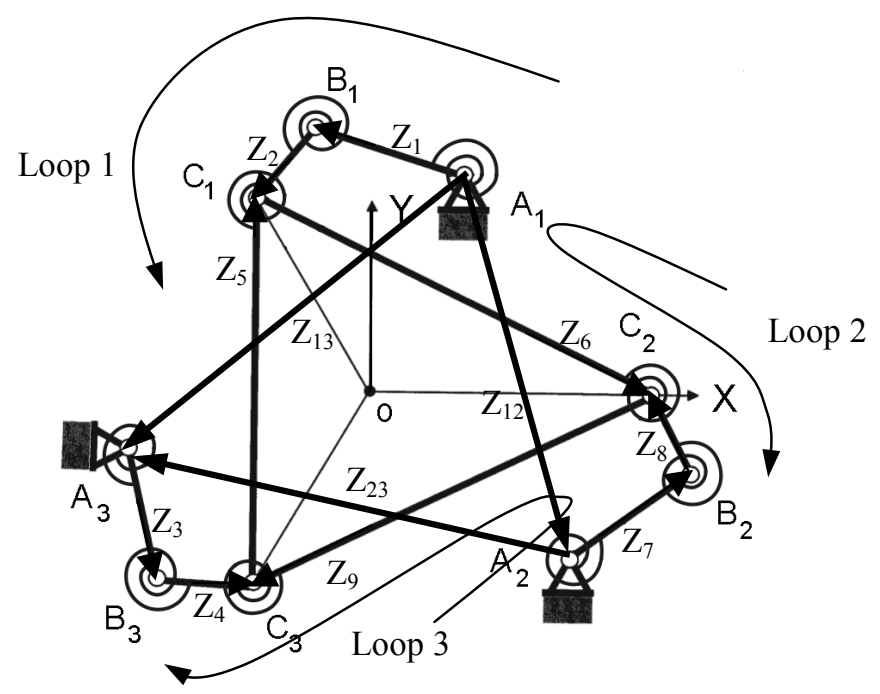

Figure 5: Three-loop 3RRR structure analysis

Loop 1:

$Z_{1}+Z_{2}=Z_{13}+Z_{3}+Z_{4}+Z_{5}$

Loop 2:

$Z_{1}+Z_{2}+Z_{6}=Z_{12}+Z_{7}+Z_{8}$

Loop3

$Z_{7}+Z_{8}+Z_{9}=Z_{23}+Z_{3}+Z_{4}$

Since the micromanipulation device moves in micro scales, $\cos \left(\Delta \theta_{i}\right) \approx 1$ and $\sin \left(\Delta \theta_{i}\right) \approx \Delta \theta_{i}$. The six equations can be simplified. 
The three input angular displacements, which are $\Delta \theta_{A i}(i=1,2,3)$, have been identified ${ }^{17}$ as below:

$$
\Delta \theta_{A i}=-\frac{\Delta l_{i}}{R_{o}}, \quad i=1,2,3
$$

where $\Delta l_{i}$ are the elongations of the PZT actuators

$R_{o}$ is the distance shown in Fig. 6.

The negative sign indicates that the direction of rotation is clockwise

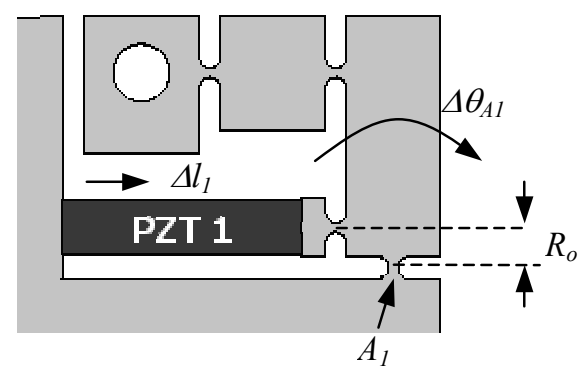

Figure 6: Driving element of the 3RRR compliant micromanipulation system

By substituting all the parameters: link lengths and the initial angular displacements of the flexure hinges, and the three input angular displacements (see Eqn. 4) into the closed-loop equations (see Eqn. 1 to 3), the six equations become linear. They can be solved simultaneously to obtain the unknown angular displacement increments without involving iterations. These unknowns are expressed in terms of the input displacements of the PZT actuators, $\Delta l_{i}(i=1,2,3)$, as shown:

$$
\begin{aligned}
& \Delta \theta_{B}=\left[\begin{array}{l}
\Delta \theta_{B 1} \\
\Delta \theta_{B 2} \\
\Delta \theta_{B 3}
\end{array}\right]=\left[\begin{array}{ccc}
481 & 320 & -190 \\
-190 & 481 & 320 \\
320 & -190 & 481
\end{array}\right]\left[\begin{array}{l}
\Delta l_{1} \\
\Delta l_{2} \\
\Delta l_{3}
\end{array}\right] \\
& \Delta \theta_{C}=\left[\begin{array}{l}
\Delta \theta_{C 1} \\
\Delta \theta_{C 2} \\
\Delta \theta_{C 3}
\end{array}\right]=\left[\begin{array}{ccc}
-255 & -380 & 130 \\
130 & -255 & -380 \\
-380 & 130 & -255
\end{array}\right]\left[\begin{array}{l}
\Delta l_{1} \\
\Delta l_{2} \\
\Delta l_{3}
\end{array}\right]
\end{aligned}
$$

By knowing all the unknown angular displacements of the flexure hinges, the forward kinematics can be derived easily. Mathematically, forward kinematics is derived to find the positions and orientations $(\Delta x, \Delta y, \Delta \gamma)^{*}$ of the end-effector when the actuated joint variables $\left(\Delta l_{1}, \Delta l_{2}, \Delta l_{3}\right)$ are given. A Jacobian matrix is normally used to relate the velocity of an end-effector to the velocity of actuators. However, for the case of micromanipulation systems, the Jacobian matrix can be defined as a matrix to relate $\left(\Delta l_{1}, \Delta l_{2}, \Delta l_{3}\right)$ with $(\Delta x, \Delta y, \Delta \gamma)^{12}$ (see Eqn. 7). The displacements of the PZT actuators are substantially small compare to the link lengths. The motions of the 3RRR mechanism are very small. Therefore, the micromanipulation device is almost configurationally invariant and its Jacobian matrix is assumed to be constant:

$$
\left[\begin{array}{l}
\Delta x \\
\Delta y \\
\Delta \gamma
\end{array}\right]=J\left[\begin{array}{l}
\Delta l_{1} \\
\Delta l_{2} \\
\Delta l_{3}
\end{array}\right] \quad \text { where } \quad J=\left[\begin{array}{ccc}
1.905 & 1.315 & -3.22 \\
2.618 & -2.96 & 0.341 \\
-59.96 & -59.96 & -59.96
\end{array}\right]
$$

\footnotetext{
${ }^{*} \Delta x$ and $\Delta y$ are the translational motions along $\mathrm{x}$ - and $\mathrm{y}$-axis respectively. $\Delta \gamma$ is the rotational motions about the $\mathrm{z}$-axis.
} 


\section{COMPARISON OF THE KINEMATIC MODELS}

A full forward kinematics of the same 3RRR compliant micromanipulation system was derived by a previous researcher ${ }^{17}$. The full kinematic model might be more accurate, however the procedures to derive the full model are more complicated compared to the linear kinematic model presented in this paper. The simulation results of the full kinematic model are compared with that of the linear kinematic model. Fig. 7 below plots the simulated positions and orientations of the end-effector for the full and the linear kinematic model. These positions and orientations are obtained when PZT 1 (see Fig. 1) is extended from 0 to 12 microns.
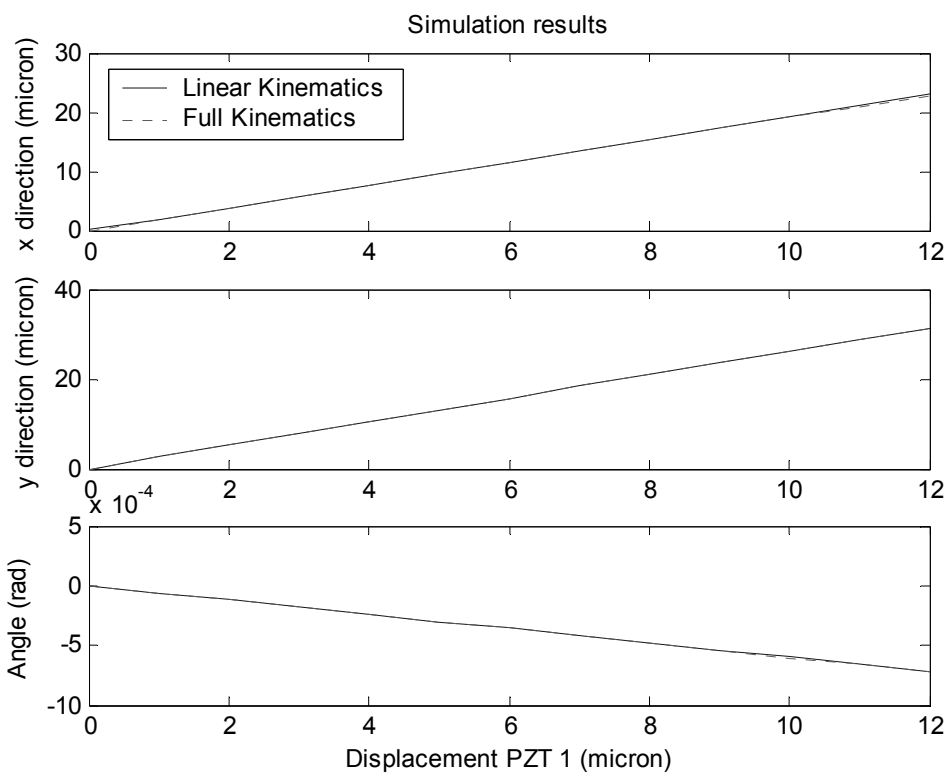

Figure 7: Results comparison of the linear and full kinematic models

\subsection{Discussions}

Table 2 exhibits the maximum errors of the positions and orientations at the end-effector using the linear kinematic model. The errors of the rotational motions about $\mathrm{z}$-axis are more sensitive than that of the translation motions along $\mathrm{x}$ and y-axis. These errors are due to the simplification of the linear kinematic model (i.e. $\cos (\Delta \theta) \approx 1$ and $\sin (\Delta \theta)$ $\approx \Delta \theta$ ). However, the errors are very small and insignificant. Hence, it can be concluded that the linear kinematic model derived in this paper is as accurate as the full kinematic model. The methods used to derive the linear kinematics are more simply and easily employed compared to the conventional methods used to derive the full model. Furthermore, the full kinematic model is non-linear, which increases the computational time and also raises the effort to achieve real-time control. For design optimisation, the linear kinematic model is more efficient, in terms of calculation time, when evaluating various design alternatives that satisfies the requirements, such as dexterity, workspace and control. The simple and linear kinematic model could also improve the ease of kinematic calibration processes; therefore the parameters in the kinematic model can be adjusted efficiently to best represent the kinematic behaviour of the 3RRR compliant mechanism.

\begin{tabular}{|c|c|c|}
\hline & \multicolumn{2}{|c|}{ Maximum Errors } \\
\hline & Units & $\%$ \\
\hline$\Delta \mathrm{x}$ & $135 \mathrm{~nm}$ & $5.9 \times 10^{-7}$ \\
\hline$\Delta \mathrm{y}$ & $24.5 \mathrm{~nm}$ & $7.8 \times 10^{-8}$ \\
\hline$\Delta \gamma$ & $2.2 \mu \mathrm{rad}$ & 0.31 \\
\hline
\end{tabular}

Table 1: Maximum errors of the positions and orientations 


\section{STATIC MODEL}

The static model of the 3RRR compliant mechanism can be derived as:

$$
F=K(\Delta l)
$$

where $F$ is a $3 \times 1$ force vector, $\Delta l$ is a $3 \times 1$ actuator displacement vector and $K(\Delta l)$ is a $3 \times 3$ stiffness matrix which depends on $\Delta l$. The stiffness matrix can be obtained by finding the potential energy of the 3RRR compliant mechanism, and by performing the partial derivative to the potential energy. The potential energy of the compliant mechanism is the elastic energy stored in the nine flexure hinges, labelled as $A_{i}, B_{i}$ and $C_{i}(i=1,2,3)$. The potential energy is:

$$
P=\frac{1}{2} K_{b}\left(\sum_{i=1}^{3} \Delta \theta_{A i}^{2}+\Delta \theta_{B i}^{2}+\Delta \theta_{C i}^{2}\right)
$$

$\Delta \theta_{A i}, \Delta \theta_{B i}$ and $\Delta \theta_{C i}$ represent the small angular displacement increments of the flexure hinges

$K_{b}$ is the spring stiffness of the flexure hinges ${ }^{20}, K_{b}=\frac{2 E b t^{5 / 2}}{9 \pi^{1 / 2}}$

where $E$ is the Young's Modulus

$b, r$ and $t$ are the dimensions of the flexure hinge, shown in Fig. 8.

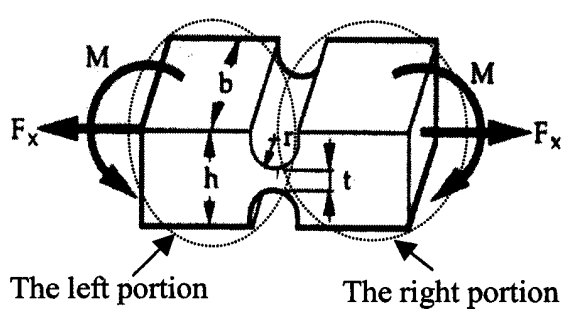

Figure 8: Dimensions of flexure hinge $\mathrm{e}^{20}$

By taking the partial derivative of Eqn. (9), the static model is obtained:

$$
F=K(\Delta l)=\left[\begin{array}{c}
\frac{\partial P}{\partial \Delta l_{1}} \\
\frac{\partial P}{\partial \Delta l_{2}} \\
\frac{\partial P}{\partial l_{3}}
\end{array}\right]=\left[\begin{array}{ccc}
0.277 \times 10^{8} & 692908 & 692908 \\
692908 & 0.277 \times 10^{8} & 692908 \\
692908 & 692908 & 0.277 \times 10^{8}
\end{array}\right]\left[\begin{array}{l}
\Delta l_{1} \\
\Delta l_{2} \\
\Delta l_{3}
\end{array}\right]
$$

For some of the micro-motion applications that operate at very low frequencies, such as biological cell manipulations, the static model in (10) can be used to describe the quasi-static state of the 3RRR compliant mechanism. This can be explained by looking at a dynamic model of the 3RRR micro-motion system. Normally, the dynamic model consists of an inertia term which is related to joint accelerations; a centrifugal/Coriolis term which is related to the joint velocities; and a stiffness term which is related to the joint displacements (see Eqn. 11). However at low operating frequencies, the velocities and accelerations of the joints are very small and insignificant. Hence, the inertia term and the centrifugal/Coriolis term are neglected. Therefore, only the stiffness term remains in the dynamic model. This dynamic model, which consists of only the stiffness term, is referred as the quasi-static model (Eqn. 10) in this paper. This quasi- 
static model has very large diagonal terms relative to non-diagonal terms, indicating that the stiffness of the $3 R R R$ compliant mechanism is almost uncoupled. This uncoupling property agrees with the experimental results ${ }^{21}$. The uncoupling property of this 3 RRR compliant mechanism will improve control efficiency tremendously ${ }^{21}$. For example, a feedforward stiffness cancellation controller ${ }^{22}$ will not be required to decouple the stiffness of a micro-motion system.

$$
Q=M(\Delta l) \Delta \ddot{l}+C(\Delta l, \Delta i) \Delta i+K(\Delta l)
$$

where $M$ is an inertia matrix, $C$ is a centrifugal/Coriolis matrix, $K$ is a stiffness matrix and $Q$ is a actuator force vector.

\section{CONCLUSIONS AND FUTURE WORK}

This paper develops a method of deriving a simple, linear and yet effective forward kinematic model of a 3RRR compliant micromanipulation device. The method is based on the loop closure theory and the concept of the pseudorigid-body model. The derived forward kinematics is linear and does not require iteration techniques to solve. Hence it is computationally efficient. The results of this linear kinematic model are compared with that of a full kinematic model. The linear model is proved to be as accurate as the full model. The static model of the 3RRR micro-motion system is found easily using the results of the derived linear kinematics. The uncoupling property, based on the static model, of the 3RRR system is discussed briefly. For future work, experiments will be conducted to further verify the accuracies of the linear kinematic model.

\section{ACKNOWLEDGEMENTS}

The authors would like to greatly acknowledge the support of the Adelaide Robotics Research Group at the University of Adelaide and the use of its facilities. Yuen Kuan Yong expresses her appreciation to the School of Mechanical Engineering for providing financial assistance.

\section{REFERENCES}

1. S. Yu and B. Nelson, “Autonomous injection of biological cells using visual servoing," Int. Symp. on Experimental Robotics (ISER), Honolulu, Hawaii, 2000.

2. L. Howell, Compliant Mechanisms, Chap.1, John Wiley \& Sons, Inc., New York, 2001.

3. A. Codourey, "Dynamic modeling of parallel robots for computed-torque control implementation," The International Journal of Robotics Research, 17(12), 1325-1336, 1998.

4. O. Ma and J. Angeles, "Direct kinematic and dynamics of a planar three-dof parallel manipulator," Advances in Design Automation, 2, 313-320, 1993.

5. J. Ryu, D. Gweon and K. Moon, "Optimal design of a flexure hinge based XY $\theta$ wafer stage," Precision Engineering, 21, 18-28, 1997.

6. P. Gao, S. Swei and Z. Yuan, "A new piezodriven precision micropositioning stage utilizing flexure hinges," Nanotechnology, 10, 394-398, 1999.

7. C. Gosselin and J. Angeles, "The optimum kinematic design of a planar three-degree-of-freedom parallel manipulator," Journal of Mechanisms, Transmissions and Automation in Design, 110, 35-41, 1988.

8. I. Her and J. C. Chang, "A linear scheme for the displacement analysis of micropositioning stages with flexure hinges," Journal of Mechanical Design, Trans. ASME, 116, 770-776, 1994.

9. M.-S. Tsai, T.-N. Shiau, Y.-J. Tsai, T.-H. Chang, "Direct kinematic analysis of a 3-RPS parallel mechanism," Mechanism and Machine Theory, 38, 71-83, 2003.

10. G. R. Dunlop and T. P. Jones, "Position anlaysis of a 3-DOF parallel manipulator," Mechanism and Machine Theory, 32 (8), 903-920, 1997.

11. F. Tahmasebi and L.W. Tsai, "Closed-form direct kinematics solution of a new parallel minimanipulator," Journal of Mechanical Design, 116, 1141-1147, 1994.

12. W. J. Zhang, J. Zou, G. Watson, W. Zhao, G. Zong and S. Bi, "Constant-Jacobian Method for Kinematics of a 3 DOF Planar Micro-Motion Stage,” Journal of Robotic Systems, 19(2), 63-79, 2002.

13. J. W. Ryu, S.-Q. Lee, D.-G. Gweon and K. S. Moon, "Inverse kinematic modeling of a coupled flexure hinge mechanism," Mechatronics, 9, 657-674, 1999. 
14. Y. Ohya, T. Arai, Y. Mae, K. Inoue and T. Tanikawa, "Development of 3-DOF Finger Module for Micro Manipulation," Proceedings of the 1999 IEEE/RSJ International Conference on Intelligent Robots and Systems, 894-899, 1999.

15. G. B. Chung, B.-J. Yi, I. H. Suh, W. K. Kim, and W. K. Chung, "Design and Analysis of a Spatial 3-DOF Micromanipulator for Tele-operation," Proceedings of the 2001 IEEE/RSJ International Conference on Intelligent Robots and Systems, Maui, Hawaii, 337-342, 2001.

16. J. Hesselbach, N. Plitea and R. Thoben, "Advanced technologies for micro assembly," Proceedings of the International Society for Optical Engineering, 3202, 178-190, 1998.

17. J. Zou, "Kinematics, Dynamics and Control of a Particular Micro-Motion System," Masters Thesis, printed by the Advanced Engineering Design Laboratory, University of Saskatchewan, 2000.

18. J. H. Shim, S. K. Song, D.S. Kwon and H.S. Cho, "Kinematic Feature Analysis of a 6-Degree-of-Freedom InParallel Maniputlator for Micro-Positioning," Proceedings of 1997 IEEE/RSJ International Conference on Intelligent Robots and Systems, Grenoble, France, 1617-1623, 1997.

19. L. L. Howell and A. Midha, "A Loop-Closure Theory for the Analysis and Synthesis of Compliant Mechanisms," Journal of Mechanical Design, 118, 121-125, 1996.

20. J. M. Paros and L. Weisbord, "How to design flexure hinges," Machine Design, 37, 151-156, 1965.

21. D.C. Handley, W.J. Zhang, T.-F. Lu and W. Zhao, "Multiple degree of freedom compliant mechanism possessing nearly uncoupled dynamics: experimental findings," Proceedings of the SPIE International Symposium on Smart Materials, Nano- and Micro-Smart Systems, Melbourne, 2002.

22. J. Speich and M. Goldfard, "A compliant-mechanism-based three degree-of-freedom manipulator for small-scale manipulation," Robotica, 18, 95-104, 2000. 